\title{
Periosteum: The forgotten source of osteoprogenitors
}

\section{Periosteo: La fuente olvidada de osteoprogenitores}

\author{
Matthew Stewart ${ }^{1}$ \\ 1 Department of Veterinary Clinical Medicine, University of Illinois, USA. Email: matt1@illinois.edu
}

\begin{abstract}
Bone is one of the few tissues capable of authentic regenerative repair. However, despite advances in surgical technique, orthopaedic hardware and our understanding of fracture biology, inadequate bone repair remains a major concern in both veterinary and human medicine. Cell-based technologies provide opportunities to utilize the osteogenic capacities of Mesenchymal Stem Cells (MSC) to augment bone repair. Much of the research on MSC biology has focused on cells derived from the bone marrow/ endosteal compartment; however, osteoprogenitor cells (OPC) also reside in the periosteum.

Periosteum develops as a fibro-cellular envelope surrounding developing skeletal elements. The inner, or cambium layer of periosteum, includes committed OPCs directly adjacent the bone surface, and a distinct sub-population of progenitors within the periosteal mid-substance that retain both chondrogenic and osteogenic capacities. During skeletogenesis, periosteal OPCs are responsible for appositional intramembranous bone formation that increases the radial diameter of long bones. Of critical importance, periosteal stem cells are the predominant cell population responsible for generating the cartilaginous or 'soft' callus that provides intermediate stabilization and a scaffold for subsequent callus ossification by endochondral ossification; the primary mechanism of bone repair.

In recent experiments using isolates from 'donor-matched' periosteum and bone marrow, we have found that the basal osteogenic capacity of equine OPCs is considerably less than that of bone marrowderived MSCs. Periosteal OPCs require exogenous Bone Morphogenetic Protein (BMP) for robust osteogenesis, a finding consistent with the clinical responses of bone to recombinant BMP protein. Perhaps more surprising, the osteogenic capacity of adult (2-10 years of age) OPCs is comparable to those of young foals', although the cell yield is considerably greater from foal specimens. In light of the vital importance of callus formation for successful fracture healing of most, further research on the biology and clinical manipulation of periosteal OPCs is highly warranted.
\end{abstract}

\section{References}

Chang, H. \& Knothe Tate, M.L. 2012. Concise review: the periosteum: Tapping into a reservoir of clinically useful progenitor cell. Stem Cells Trans. Med. 1(6): 480-491. DOI: 10.5966/sctm.2011-0056. 
Ciencias

Veterinarias

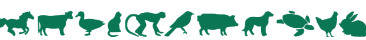

Rev. Ciencias Veterinarias, Vol. 37, Nº 3, [22-23], E-ISSN: 2215-4507

First Symposium on Regenerative Medicine and Nanotechnology, Costa Rica 2019 DOI: https://doi.org/10.15359/rcv.37-3.7

URL: http://www.revistas.una.ac.cr/index.php/veterinaria/index

Colnot, C. 2009. Skeletal cell fate decisions within periosteum and bone marrow during bone regeneration. J. Bone Miner. Res. 24(2): 274-282. DOI: 10.1359/jbmr.081003

Dawson, L.A., Simkin, J., Sauque, M., Pela, M., Palkowski, T. \& Muneoka, K. 2014. Analogous cellular contribution and healing mechanisms following digit amputation and phalangeal fracture in mice. Regeneration 3(1): 39-51. DOI: 10.1002/reg2.51

Wang, T., Zhang, X., Bikle, D.D. 2017. Osteogenic differentiation of periosteal cells during fracture healing. J. Cell Physiol. 232(5): 913-921. DOI: 10.1002/jcp.25641 